\title{
Views of First-Time Expectant Mothers on Breastfeeding: A Study in Three Health Facilities in Accra, Ghana
}

\author{
Freda Intiful, Claudia Osei, Rebecca Steele-Dadzie, Ruth Nyarko, and Matilda Asante \\ School of Biomedical and Allied Health Sciences, Department of Nutrition and Dietetics, University of Ghana, Accra, Ghana \\ Correspondence should be addressed to Freda Intiful; fdintiful@chs.edu.gh
}

Received 13 April 2017; Revised 10 August 2017; Accepted 7 September 2017; Published 19 October 2017

Academic Editor: Carol J. Burns

Copyright (C) 2017 Freda Intiful et al. This is an open access article distributed under the Creative Commons Attribution License, which permits unrestricted use, distribution, and reproduction in any medium, provided the original work is properly cited.

\begin{abstract}
The objective of this study was to evaluate the views of first-time expectant mothers on breastfeeding. A qualitative study approach using focus group discussions was used to solicit the views of 25 expectant first-time mothers. The results indicated the intention to breastfeed, though some were willing to opt for formula feeding when the need arises. Knowledge on breastfeeding issues was minimal among this group. Common sources of information on breastfeeding issues were obtained from home (relatives), hospital, and television. The need to support and provide adequate education on breastfeeding issues is critical among this category of women.
\end{abstract}

\section{Introduction}

The option to breastfeed or not is influenced by several factors including but not limited to cultural practices, health status of both mother and infant, nutrition knowledge, and support from family and other stakeholders. First-time expectant mothers may have several perceptions about breastfeeding based on what they have seen or heard from people about breastfeeding. Most of them may feel unskilled and therefore are unable to decide on feeding options while others may feel confident and therefore can decide on feeding options in the early stages of pregnancy [1].

In spite of the numerous established benefits of breastfeeding, feeding infants with baby formula has also gained a lot of recognition among first-time mothers. As a global public health strategy, the World Health Organization (WHO) recommends exclusive breastfeeding for the first six months as the gold standard for the feeding of infants during the first six months of life [2]. Some of the reported benefits of exclusive breastfeeding are its ability to reduce childhood morbidity and mortality [3-5]. Studies that were conducted in Denmark, Honduras, and New Zealand also demonstrated the effect of breastfeeding on intellectual and motor development of children [6-8]. The ability of breastfeeding to be protective against the development of chronic conditions such as obesity and diabetes has been reported elsewhere. For example, it was reported that breastfed infants were more than $30 \%$ less likely to be obese later in life and also had about $33 \%$ increased risk of developing diabetes if not breastfed by the time of discharge from the hospital [9-11]. The economic benefits cannot be overemphasized especially in a developing economy like Ghana [12].

Many first-time expectant mothers may have the intention to breastfeed; however challenges during delivery may cause them to resort to other forms of feeding [13]. The decision to breastfeed could also be enhanced by the support they receive from family and other stakeholders [14].

In the most recent reports, Ghana has experienced reduced rates of exclusive breastfeeding from $63 \%$ in 2008 to $52 \%$ in $2013[15,16]$ (the 2013 Ghana Demographic Health Survey has the most current national data on exclusive breastfeeding rate in Ghana). Considering many benefits of breastfeeding to both mother and baby, it is crucial to address the barriers that prevent mothers from breastfeeding. If the first-time mother gets it right, then the likelihood of breastfeeding all subsequent children is high. Therefore, it is important to identify the barriers and beliefs in order to effectively address them. This qualitative study was conducted to evaluate the views of first-time expectant mothers on breastfeeding. 
TABLE 1: Background characteristics of expectant mothers.

\begin{tabular}{|c|c|c|}
\hline Characteristics & Frequency $(n)$ & Percentage $(\%)$ \\
\hline \multicolumn{3}{|c|}{ Age (mean age of participant $24.71 \pm 6.18$ ) } \\
\hline$\leq 25$ & 12 & 48 \\
\hline $26-40$ & 11 & 44 \\
\hline$>40$ & 2 & 8 \\
\hline \multicolumn{3}{|l|}{ Religion } \\
\hline Christian & 21 & 84 \\
\hline Muslim & 4 & 16 \\
\hline \multicolumn{3}{|l|}{ Marital status } \\
\hline Married & 15 & 60 \\
\hline Single & 10 & 40 \\
\hline \multicolumn{3}{|l|}{ Employment status } \\
\hline Employed & 18 & 72 \\
\hline Unemployed & 7 & 28 \\
\hline \multicolumn{3}{|l|}{ Educational level } \\
\hline No education & 4 & 16 \\
\hline Primary & 3 & 12 \\
\hline Junior high school & 4 & 16 \\
\hline Senior high school & 9 & 36 \\
\hline Tertiary & 5 & 20 \\
\hline
\end{tabular}

\section{Methodology}

2.1. Study Site and Design. A qualitative study design involving focus group discussions was employed. The study was conducted in three health facilities (Kaneshie Polyclinic, Mamprobi Polyclinic, and Ussher Polyclinic). All three hospitals are located in the Accra Metropolis of the Greater Accra Region of Ghana. These three hospitals are located at areas with similar socioeconomic characterizations. The inhabitants of these areas mainly belong to the low and middle class. The main socioeconomic activity in these three areas is trading but there are those who also engage in whitecollar jobs. Two major markets (Kaneshie Market Complex and Makola Market) are all situated within the catchment areas.

2.2. Participants. Twenty-five (25) first-time pregnant women participated in this study. They were made up of women between the ages of 18 and 46 years who had not delivered live babies or breastfed before. They were recruited from antenatal clinics at the various polyclinics. First-time pregnant women who came for antenatal for the first time were purposefully introduced to the researcher by the nurses. The researcher further explained the aim and procedures of the study to them. Those who consented to the study were recruited into the study.

2.3. Data Collection. A structured questionnaire was used to obtain information on the background of the expectant mothers. Three (3) different focus group discussions were held at the three (3) respective polyclinics. A facilitator moderated the discussions with the help of an assistant who took note of other relevant observations. The moderator used open ended questions to solicit views of expectant mothers on breastfeeding.

The discussions were held in Twi (a popular Akan dialect in Ghana) and recorded because most of the participants could not express themselves well in English. The discussions were then transcribed and translated verbatim into English. To reduce the effect to loss of meaning, the researcher moderated the translation process in collaboration with a translator to ensure that the intended meaning is maintained. Data were inputted into Microsoft word. Data were further coded manually by two of the researchers (FI and CO). Variations in coding were discussed and resolved. Agreed codes were categorized and developed into themes. These were further analyzed and discussed with reference to other relevant and current literatures.

2.4. Ethical Considerations. Ethical approval for this study was granted by the Ethics and Protocol Review Committee of the School of Biomedical and Allied Health Sciences, University of Ghana. The purpose and protocol of the study were explained to the participants and a written consent was obtained to ensure that participation was fully voluntary. Participants in the study were identified with pseudo names and therefore could not be linked with their comments.

\section{Results}

The mean age of the participants was $24.71 \pm 6.18$ years. Majority of the participants were Christians (84\%). More than half $(60 \%)$ of the participants were married. The employment rate among participants was $72 \%$. Most of the participants had some form of education (Table 1). 
The results from the focus group discussions were presented under the following themes; reactions of participants to the realization of being pregnant; feeding plan of first-time mothers; knowledge on breastfeeding; benefits of breastfeeding; views on formula feeding; other feeding options in case the mother cannot breastfeed; sources of information on breastfeeding; key people who may influence their breastfeeding; people and places for assistance during breastfeeding.

3.1. Reaction of Participants to the Realization of Being Pregnant. The reactions of the participants to the news that they were expecting their first child were varied. Some were genuinely excited about their first child, others were not excited at all, and others, though were not planned, decided to accept their fate and cope with the situation. Here are some of the varied responses:

I was very happy because I did not know that at my age God would remember me. [with so much passion] (46 year old woman)

I was kind of disappointed because I was not prepared. (25 year old woman)

I was happy. [shrugging her shoulders]. (18 year old woman)

3.2. Feeding Plan of First-Time Mothers. Most of the respondents chose the option to breastfeed. Some however opted for formula feeding. One mother stated that

I will breastfeed for the first four months and after that I will introduce porridges to the child because I think by 4 months the breastmilk will not satisfy the child, so I will add other foods to the breastmilk. (25 year old mother)

Another woman also indicated that she will rather feed her baby formula.

I will give formula food to the baby. (18 year old woman)

3.3. Knowledge on Breastfeeding Issues. Some of the respondents were familiar with the issues of breastfeeding. Others were not too conversant with the benefits of breastfeeding. Those who knew about the benefits of breastfeeding could explain in detail indicating that they had some education on breastfeeding. One woman commented that "breastmilk makes the child look nice and healthy and also strong." Others were also of the view that breastmilk improves the child's intelligence. In this regard a woman said that "breastmilk opens the mind of the child" (28 year old woman), literally meaning that the child becomes more intelligent. There was also the opinion to breastfeed no matter the circumstance. This was well expressed in the opinion of one woman:

When you give birth to a child, you need to breastfeed the baby because it has all the necessary nutrients for the child to grow. Unless the child's mum is dead, the child would not be breastfed but so far as the mother is alive, the child should be breastfed. (25 year old woman)

3.4. Benefits of Breastfeeding. When the women were asked about the benefits of breastfeeding to the mother and the baby, it appeared that they were more conversant with the benefits to the child than to the mother. The responses of the benefits included protection of the child from diseases, making the child strong, the ability of the mother to save some money, and also reducing the stress of having to visit the hospital periodically due to frequent illness of the baby. The following are some of the responses from the mothers:

Well, what people have said and I have heard is that if you do not breastfeed, the breastmilk fills the breast and makes you uncomfortable and it is painful, so when you breastfeed, it helps you the mother feel comfortable. (32 year old woman)

When you breastfeed, it helps you the mother save money and does not make your breast fill up and become painful. (26 year old woman)

When you breastfeed the child, it makes the child more healthy than giving formula food, makes the child stronger and protects the child too from diseases. (23 year old woman)

It helps prevent other health issues from arising and for your own good you have to breastfeed your child because it helps the child from getting health issues. It also saves you from the stress of going up and down to the hospital in case your child gets sick. (25 year woman)

3.5. Views on Formula Feeding. There were varied views on formula feeding. Some expressed strong reservations with the use of formula to feed the newborn baby.

This is from the belief that the formula may not be safe because it is in a tin. Others also believed that the formula is full of chemicals therefore not appropriate for the baby. These were expressed in the sentiments of some of the women:

Some of the children cannot tolerate formula food but with the natural foods they can eat it very well. Also with the formula foods, there are a lot of chemicals in it. So I would give the natural foods than the formula food. (34 year old woman)

I think because it is a processed food it is not $100 \%$ safe. Also, because it is in a tin, it can rust as compared to breastmilk that we know is safe. (25 year old woman)

As I said earlier on, these formula foods have chemicals in it and it is not healthy for your baby. So I would not give it at all. It also contains too much sugar from my sister's experience. (29 year old woman) 
Some of the women were also of the view that circumstances such as the unavailability of the mother as a result of her work schedule could necessitate the introduction of formula. For example, one woman commented, "It depends on the job you are doing. So maybe during the week, I will give formula food but on weekends, I will prepare both formula food and breastmilk and will give any of which the child prefers." (24 year old woman)

Another woman also stated that "I think it is good because if you are not around the child can still eat. Also, what you the mother is lacking in breastmilk, you can get it from formula foods." (22 year old woman)

One other woman was strongly in support of formula feeding since she claims her friend used that option and found it to be very beneficial: "It is good because I have a friend who gave her child formula food and the child is healthy, so formula food is good." (19 year old woman)

3.6. Alternative Feeding Options When Mother Cannot Breastfeed. In the event of not being able to breastfeed, the women indicated various feeding options they would use. Feeding the child "koko" (fermented maize porridge) was the main choice for most of the women. Others could not give a definite answer as they had not thought about it before. Some also mentioned that they would give their baby formula food because it is close to the nature of breastmilk. The responses of the women allude to their preferences:

\section{I will give my child porridge because formula foods are not good for the child's health, so instead I will give the child our local foods, mill it and put it down for the child to eat. (29 year old mother) \\ I will give my child food that is close to the breastmilk or any light food. (28 year old)}

3.7. Source of Information and Key People Who Influence Breastfeeding. The women indicated that their main sources of information on breastfeeding were from the hospital, school, at home, and health talks on TV shows.

The women were asked key people they think would most likely influence their breastfeeding adherence. They were unanimous in stating that they would listen to their mothers and what is said in the hospital. Here is a response:

I would listen to my mother and the hospital. (34 year old woman)

3.8. People and Places for Assistance during Breastfeeding. When they were further asked who they will go to for assistance when in challenges with regard to breastfeeding, most participants mentioned their mothers and the hospitals. Here are some responses:

From my mother, because she has given birth before and I am now about to. (25 year old woman)

From the hospital because they can show you how to feed the baby well than the house. (19 year old woman)

\section{Discussion}

This study aimed at providing more information to support the existing literature on breastfeeding. The qualitative nature of the study provides in-depth information concerning the views of expectant first-time mothers. The findings of this study will provide healthcare professionals, policy makers, and health program promoters information to help develop better support systems especially for expectant first-time mothers and know how to disseminate information to the mothers during their antenatal visits and the general public. This particular group was targeted because their views may be carried into the next phase of child care. In cases where these views become misconceptions, it may affect child care [17].

From this study, some expectant mothers expressed happiness on realization that they were pregnant while others were disappointed. Being happy about being pregnant is more likely to imply that they wanted the pregnancy. This is likely to impact on the decision of the expectant mother choosing to breastfeed as has been reported in another study in the US. The study observed that women with unwanted pregnancies were found less likely to breastfeed when compared to those who planned their pregnancies [18].

Breastfeeding is declining in Ghana. The challenge however is when it has to be done exclusively for the first six months. From the focus group discussions, it was realized that though they may opt to breastfeed their babies as advised during antenatal care sessions, the duration of the breastfeeding for the first six months was not certain. This can be attributed to the challenges they envisage that could hinder the ability to exclusively breastfeed. Some of these challenges are fuelled by perceptions and myths about breastfeeding. These myths and perceptions include the belief that breastmilk milk becomes polluted when the mother becomes pregnant while still lactating, colostrum being regarded as dirty, the fear of the lactating mother dying because of prolong breastfeeding, and the perception that breastmilk alone is not sufficient for the baby $[19,20]$. Grandmothers, especially the mothers-in-law of breastfeeding women, are significant persons who can influence what to feed the newborn baby [21]. In addition the employment status of the breastfeeding mother can be a problem. For example among city dwelling professional Ghanaian women, exclusive breastfeeding rate was $10.3 \%$ in spite of the fact that $99 \%$ were aware of exclusive breastfeeding and 91\% initiating breastfeeding within the first hour after birth [22]. Some cultural practices such as the giving of water, gripe water, and local herbs also prevent them from reaching the full six months of exclusivity [21].

Furthermore, the knowledge of the first-time mothers concerning breastfeeding had some gaps. Some of the expectant first-time mothers were conversant with some of the importance of breastfeeding to the baby. However, knowledge of the benefits of breastfeeding to the mother was minimal. This could be as a result of emphasis laid on the benefits of breastfeeding to the baby during educational sections to be more as compared to that of the mother. This calls for more thorough educational sections for women. 
The views concerning formula feeding revealed that some mothers were not in favour of the use of formula foods as they deemed it unsafe and not healthy for their babies. During antenatal care sessions, the health professionals in charge such as the midwives and the nurses counsel the mothers on the advantages of breastfeeding over the use of formula. Concerns have also been raised about formula feeding that it may be contaminated with pathogens and chemicals and that it requires adequate hygiene in its preparation [23-25].

Research has shown that it is common for first-time mothers to have delayed lactogenesis [26]. However, the views of the expectant first-time mothers during the discussion reflected a level of nescience. This may be due to the fact that, during antenatal care, more emphasis is placed on the need to breastfeed the child leaving other areas unturned. It is imperative to get mothers equipped with the knowledge on what to do when they face challenges in breastfeeding. Support and education on what to do in cases of delayed lactogenesis may be minimal. This is evident in their responses as some opted to give porridges or food close to breastmilk. They may have an idea about what to do but not much education has been done concerning this. This brings to the fore the low level of support systems or groups to help mothers who want to exclusively breastfeed as has also been reported in the UK [27].

Regarding the source of information on breastfeeding, expectant first-time mothers had varied sources of information. This is indicative of the fact that education on breastfeeding was not only limited to the hospital. Similar findings were reported in an Australian study. Their main sources of information were from healthcare professionals, relatives/friends, television, and the Internet [28]. In this present study, key people who will be more likely to influence participants' breastfeeding were their mothers (i.e., grandmothers) and health professionals. These were the same people they would consult for assistance. In a systemative review of studies that investigated the role of grandmothers in both developing and developed countries, it became apparent that grandmothers had important influence on exclusive breastfeeding (Negin 2016). Support from healthcare professionals also strengthens the self-esteem of mothers, thereby encouraging them to exclusively breastfeed [29]. It is therefore crucial that these categories of people are well educated and equipped with the issues of breastfeeding so they can impact the mothers appropriately.

\section{Conclusion}

Breast feeding was the most preferred choice of feeding for the babies of the expectant mothers studied. The mothers were generally aware of the benefits of breastfeeding especially for their babies. There was a general disapproval for the use of formula to feed babies. The expectant mothers also indicated that they will solicit for help from their mothers (i.e., grandmothers) or health professionals when they are faced with challenges regarding breastfeeding.

It is recommended that further studies comprised of an educational component on breastfeeding and a subsequent evaluation analysis would be necessary to investigate how these views were put into action after they had delivered their babies. More detailed antenatal care sessions specific for expectant first-time mothers as well as strong support systems that encourage the education on breastfeeding should be done.

\section{Conflicts of Interest}

The authors declare no conflicts of interest.

\section{Acknowledgments}

The authors wish to express their sincere thanks to all expectant mothers who volunteered to be part of this study and the staff of the antenatal clinics at the three polyclinics where the study was conducted.

\section{References}

[1] A. M. Stuebe and E. B. Schwarz, "The risks and benefits of infant feeding practices for women and their children," Journal of Perinatology, vol. 30, no. 3, pp. 155-162, 2010.

[2] Global Strategy for Infant And Young Child Feeding, WHO and UNICEF, Geneva, Switzerland, 2003.

[3] J. Clemens et al., "Early initiation of breastfeeding and the risk of infant diarrhea in rural Egypt," Pediatrics, vol. 104, no. 1, pp. e3-e3, 1999.

[4] M. Meremikwu, A. Asindi, and O. Antia-Obong, "The influence of breast feeding on the occurrence of dysentery, persistent diarrhoea and malnutrition among Nigerian children with diarrhoea," West African journal of medicine, vol. 16, no. 1, pp. 20-23, 1997.

[5] A. P. Betrán, M. De Onís, J. A. Lauer, and J. Villar, "Ecological study of effect of breast feeding on infant mortality in Latin America," British Medical Journal, vol. 323, no. 7308, p. 303, 2001.

[6] E. L. Mortensen et al., "The association between duration of breastfeeding and adult intelligence," Jama, vol. 287, no. 18, pp. 2365-2371, 2002.

[7] K. G. Dewey et al., "Effects of exclusive breastfeeding for four versus six months on maternal nutritional status and infant motor development: results of two randomized trials in Honduras," The Journal of Nutrition, vol. 131, no. 2, pp. 262-267, 2001.

[8] L. Horwood, B. Darlow, and N. Mogridge, "Breast milk feeding and cognitive ability at 7-8 years," Archives of Disease in Childhood-Fetal and Neonatal Edition, vol. 84, no. 1, pp. F23F27, 2001.

[9] M. W. Gillman et al., "Risk of overweight among adolescents who were breastfed as infants," Jama, vol. 285, no. 19, pp. 24612467, 2001.

[10] M. L. Hediger et al., "Association between infant breastfeeding and overweight in young children," Jama, vol. 285, no. 19, pp. 2453-2460, 2001.

[11] M. E. Jones, A. J. Swerdlow, L. E. Gill, and M. J. Goldacre, "Prenatal and early life risk factors for childhood onset diabetes mellitus: A record linkage study," International Journal of Epidemiology, vol. 27, no. 3, pp. 444-449, 1998.

[12] A. Kuma, "Economic and health benefits of breastfeeding: a review," Food Science and Quality Management, vol. 45, no. 39, 2015. 
[13] J. Panczuk, S. Unger, D. O'Connor, and S. K. Lee, "Human donor milk for the vulnerable infant: A Canadian perspective," International Breastfeeding Journal, vol. 9, no. 1, article no. 4, 2014.

[14] M.-T. Tarkka, M. Paunonen, and P. Laippala, "What contributes to breastfeeding success after childbirth in a maternity ward in Finland?" Women and Birth, vol. 25, no. 3, pp. 175-181, 1998.

[15] G. S. S. GSS, "Ghana Demographic Health Survey 2008," in Proceedings of the Ghana Health Service (GHS), and ICF International'08, Demographic and Health, 2008.

[16] G. S. S. GSS, "Demographic Health Survey 2014," in Proceedings of the Ghana Health Service (GHS), and ICF International, Demographic and Health, 2015.

[17] A. Freund, Expectations and experiences of first-time mothers [M.S. thesis], faculty of the University Graduate School in partial fulfillment of the requirements for the degree Master of Arts in the Department of Sociology, Indiana University, 2008.

[18] T. D. Dye, M. A. Wojtowycz, R. H. Aubry, J. Quade, and H. Kilburn, "Unintended pregnancy and breast-feeding behavior," American Journal of Public Health, vol. 87, no. 10, pp. 1709-1711, 1997.

[19] A. K.-A. Diji, V. Bam, E. Asante, A. Y. Lomotey, S. Yeboah, and H. A. Owusu, "Challenges and predictors of exclusive breastfeeding among mothers attending the child welfare clinic at a regional hospital in Ghana: A descriptive cross-sectional study," International Breastfeeding Journal, vol. 12, no. 1, article no. 13, 2017.

[20] A. Ayawine and K. A. Ae-Ngibise, "Determinants of exclusive breastfeeding: a study of two sub-districts in the Atwima Nwabiagya District of Ghana," Pan African Medical Journal, vol. 22, p. 248, 2015.

[21] R. A. Aborigo, C. A. Moyer, S. Rominski et al., "Infant nutrition in the first seven days of life in rural northern Ghana," $B M C$ Pregnancy and Childbirth, vol. 12, article no. 76, 2012.

[22] E. J. Dun-Dery and A. K. Laar, "Exclusive breastfeeding among city-dwelling professional working mothers in Ghana," International Breastfeeding Journal, vol. 11, no. 1, article no. 23, 2016.

[23] K. D. Gribble and B. L. Hausman, "Milk sharing and formula feeding: Infant feeding risks in comparative perspective?" Australasian Medical Journal, vol. 5, no. 5, pp. 275-283, 2012.

[24] C. Siew et al., "Assessing a potential risk factor for enamel fluorosis: a preliminary evaluation of fluoride content in infant formulas," The Journal of the American Dental Association, vol. 140, no. 10, pp. 1228-1236, 2009.

[25] J. G. Schier, A. F. Wolkin, L. Valentin-Blasini et al., "Perchlorate exposure from infant formula and comparisons with the perchlorate reference dose," Journal of Exposure Science and Environmental Epidemiology, vol. 20, no. 3, pp. 281-287, 2010.

[26] L. A. Nommsen-Rivers, C. J. Chantry, J. M. Peerson, R. J. Cohen, and K. G. Dewey, "Delayed onset of lactogenesis among first-time mothers is related to maternal obesity and factors associated with ineffective breastfeeding," American Journal of Clinical Nutrition, vol. 92, no. 3, pp. 574-584, 2010.

[27] A. Grant, K. McEwan, S. Tedstone, G. Greene, L. Copeland et al., "Availability of breastfeeding peer support in the United Kingdom: A cross-sectional study," Maternal and Child Nutrition, 2017.

[28] R. Newby et al., "Antenatal information sources for maternal and infant diet," Breastfeeding Review, vol. 23, no. 2, p. 13, 2015.

[29] N. Alianmoghaddam, S. Phibbs, and C. Benn, "Resistance to breastfeeding: A Foucauldian analysis of breastfeeding support from health professionals," Women Birth, 2017. 


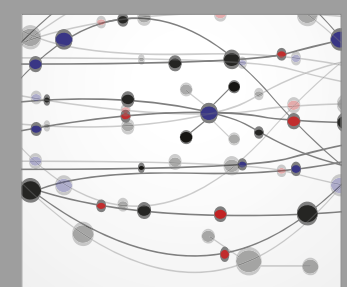

The Scientific World Journal
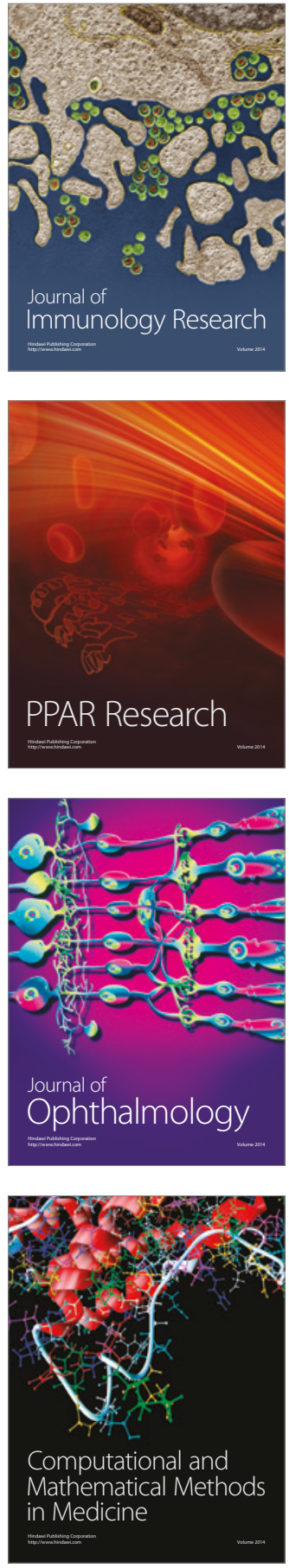

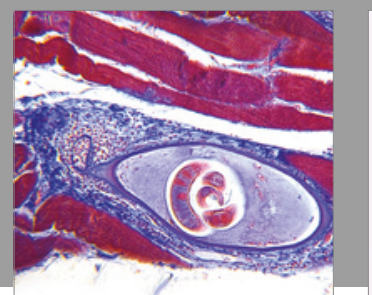

Gastroenterology Research and Practice
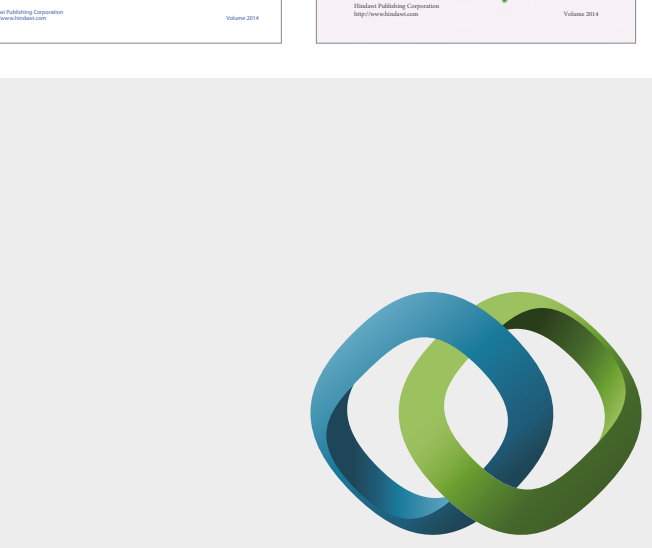

\section{Hindawi}

Submit your manuscripts at

https://www.hindawi.com
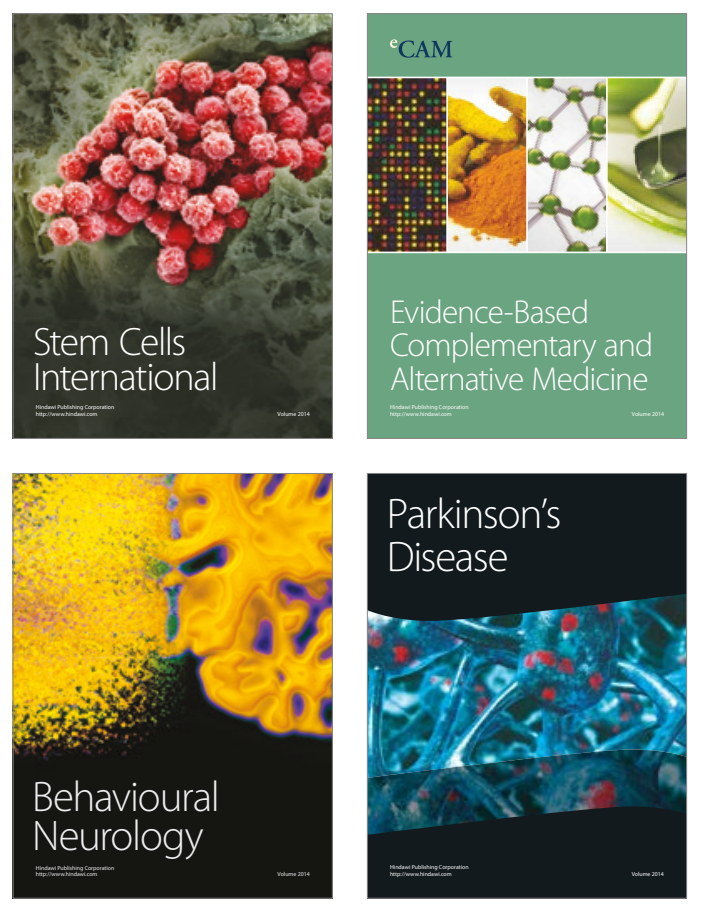
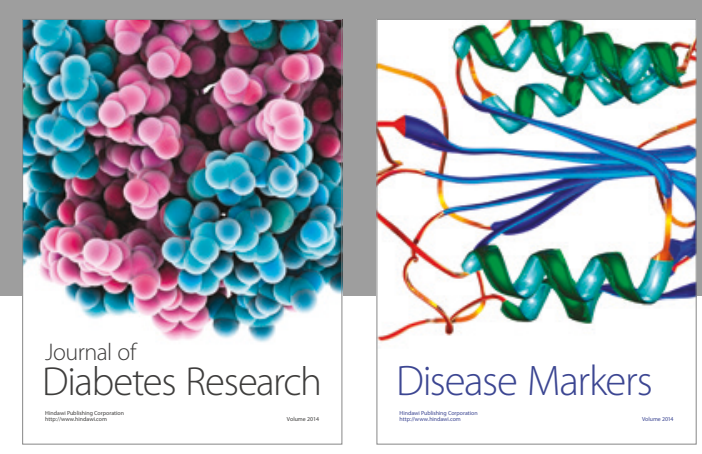

Disease Markers
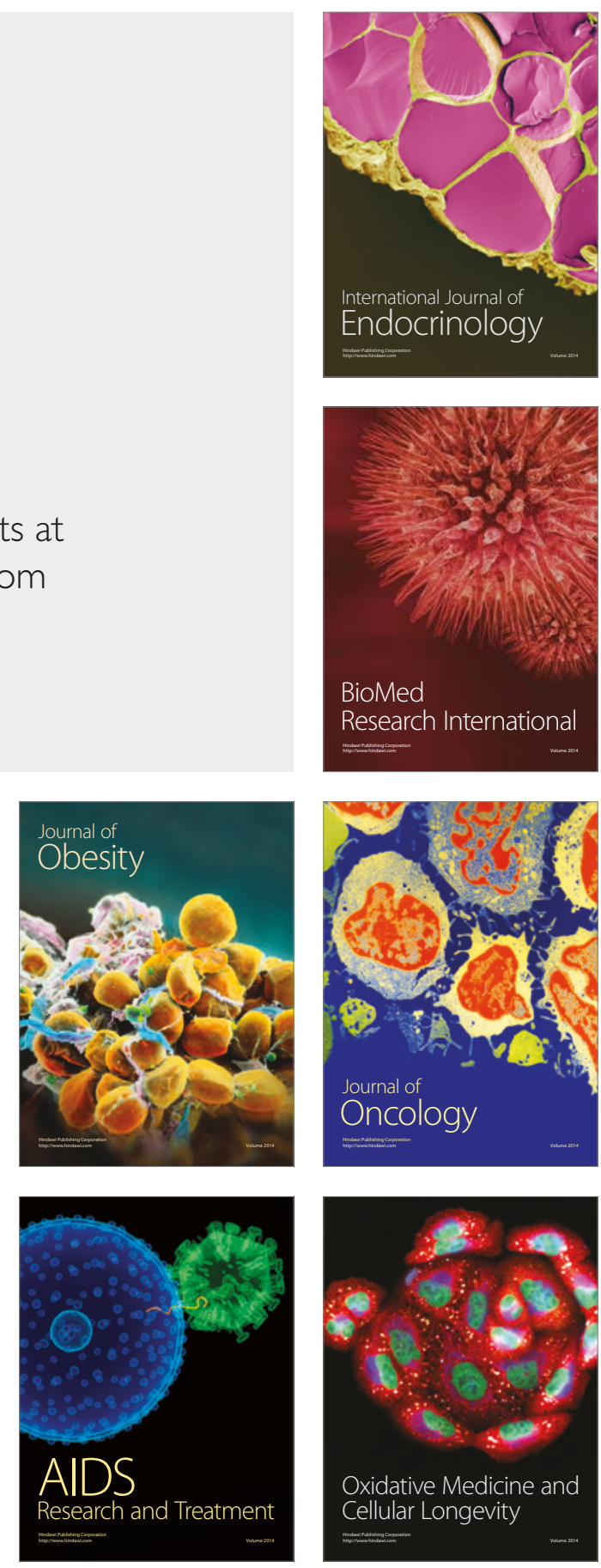